VOLUME 28 (2020) 110-126

DOI: $10.24330 /$ ieja. 768190

\title{
ON CONNECTEDNESS AND COMPLETENESS OF CAYLEY DIGRAPHS OF TRANSFORMATION SEMIGROUPS WITH FIXED SETS
}

\author{
Nuttawoot Nupo and Chollawat Pookpienlert \\ Received: 20 September 2019; Revised: 3 June 2020; Accepted: 16 June 2020 \\ Communicated by A. Çiğdem Özcan \\ Abstract. Let $\operatorname{Fix}(X, Y)$ be a semigroup of full transformations on a set $X$ \\ in which elements in a nonempty subset $Y$ of $X$ are fixed. In this paper, we \\ construct the Cayley digraphs of $\operatorname{Fix}(X, Y)$ and study some structural proper- \\ ties of such digraphs such as the connectedness and the completeness. Further, \\ some prominent results of Cayley digraphs of $\operatorname{Fix}(X, Y)$ relative to minimal \\ idempotents are verified. In addition, the characterization of an equivalence \\ digraph of the Cayley digraph of $\operatorname{Fix}(X, Y)$ is also investigated.
}

Mathematics Subject Classification (2020): 05C20, 05C25, 20M20

Keywords: Cayley digraphs of transformation semigroups, connectedness, completeness, minimal idempotents, equivalence digraphs

\section{Introduction}

In algebraic graph theory, the structures of algebraic methods are studied and then applied to problems about graphs. An interesting topic is to study properties of graphs in connection to algebraic systems. A well-known connection between graphs and algebraic systems is the construction of Cayley graphs of groups. The Cayley graph was first introduced for finite groups by Arthur Cayley in 1878. This concept was considered to explain the structures of abstract groups which are described by the set of group generators. Furthermore, the construction of Cayley graphs is also applied to semigroups. As the fact that Cayley graphs of semigroups can reflect the structural properties of semigroups, such semigroups can be visualized by constructing their Cayley graphs. For introducing the definition of the Cayley graph, let $S$ be a semigroup and $A$ a subset of $S$. The Cayley graph $\operatorname{Cay}(S, A)$ of a semigroup $S$ with respect to $A$ is defined to be a digraph with vertex set $S$ and arc set consisting of ordered pairs $(x, x a)$ for some $a \in A$ and $x$ is an arbitrary element in $S$. The set $A$ is called a connection set of $\operatorname{Cay}(S, A)$. It is easily visible that if $A$ is an empty set, then $\operatorname{Cay}(S, A)$ is considered to be an empty graph. Thus throughout this paper, the connection set $A$ will be nonempty. The Cayley 
graphs of semigroups have received serious attention in the literature. A lot of work has been done on the study of Cayley graphs of semigroups with respect to their graph theoretical properties. Many results of Cayley graphs of particular types of semigroups have been investigated. In 2006, Kelarev [9] described all inverse semigroups with Cayley graphs which are disjoint unions of complete graphs. In 2007, Fan and Zeng [5] obtained a complete description of all vertex-transitive Cayley graphs of bands. Later in 2010, Hao and Luo [6] investigated the basic structures and properties of Cayley graphs of left groups and right groups. In the same year, Khosravi and Mahmoudi [10] characterized the Cayley graphs of rectangular groups and studied their vertex-transitivity. Further in 2011, Luo, Hao and Clarke [11] considered Cayley graphs of completely simple semigroups. In addition, they studied some structural properties such as the completeness and strongly connected bipartite Cayley graphs. Indeed, it turns out that Cayley graphs of semigroups are significant not only in semigroup theory, but also in constructions of various interesting types of graphs with nice combinatorial properties. Several prominent properties of those graph constructions have been presented in numerous journals. In 2015, Suksumran and Panma [16] proposed some concepts on connected Cayley graphs of semigroups. Later in 2016, Afkhami et al. [1] constructed a new class of Cayley graphs and studied their structural properties similar to the research presented by Sinha and Sharma [15] in the same year. Furthermore, in 2018, Panda and Krishna [12] investigated the connectedness of power graphs of finite groups.

For the part of semigroup theory, one of well-known semigroups that plays a crucial role in the study of semigroups is a transformation semigroup. In group theory, the well-known theorem called Cayley's theorem asserts that any group $G$ is isomorphic to a subgroup of the symmetric group of the set $G$. Also in semigroup theory, an analogue of Cayley's theorem shows that any semigroup can be realized as a transformation semigroup of certain appropriate set. This would be a general result why the transformation semigroup is interesting to study. Some prominent results of Cayley graphs of transformation semigroups have been obtained. For instance, in 2017, Tisklang and Panma [17] investigated the connectedness of Cayley graphs of finite transformation semigroups with restricted range. Moreover, Riyas and Geetha [14], in 2018, studied the Cayley graphs of full transformation semigroups relative to the sets of idempotents. They also provided the existence of Hamiltonian cycles in such Cayley graphs. Our purpose is to study some structural properties of Cayley graphs of certain transformation semigroups. Let $X$ be a set and $Y$ a nonempty subset of $X$. Further, let $T(X)$ denote the semigroup of 
transformations from $X$ into itself under the composition of maps which is generally called the full transformation semigroup. Define the transformation semigroup $\operatorname{Fix}(X, Y)$ with a fixed set $Y$ as follows:

$$
\operatorname{Fix}(X, Y)=\{\alpha \in T(X): a \alpha=a \text { for all } a \in Y\} .
$$

Then $\operatorname{Fix}(X, Y)$ is a subsemigroup of $T(X)$. Virtually, whenever $Y$ has only one element, say $a$, the semigroup we consider is the set of all self-maps on $X$ having $a$ as their only common fixed point which is the one of interesting topics studied in fixed point theory.

The semigroup Fix $(X, Y)$ was first introduced by Honyam and Sanwong [7] in 2013. They characterized the regularity, Green's relations and ideals of $\operatorname{Fix}(X, Y)$. Later in 2016, Chaiya, Honyam and Sanwong [2] presented the characterization of the natural partial order on $\operatorname{Fix}(X, Y)$.

Here, we shall investigate certain classes of Cayley graphs of $\operatorname{Fix}(X, Y)$ such as connected digraphs, complete digraphs and equivalence digraphs. Some useful notations and relevant terminologies related to this paper will be provided in the next section.

\section{Preliminaries and notations}

In this section, some basic preliminaries and relevant notations used in what follows on digraphs, semigroups and Cayley graphs of semigroups are described. For more information, we refer to [13] on digraphs and to $[3,4,8]$ on semigroups. All sets mentioned in this paper are assumed to be finite.

A digraph $D$ (directed graph) is a pair $(V(D), E(D))$ where $V(D)$ is a nonempty set, called a vertex set, whose elements are called the vertices and $E(D)$ is the subset of the set of ordered pairs of elements of $V(D)$. In other words, the set $E(D)$ can be considered as a relation on the set $V(D)$. The elements of $E(D)$ are called the arcs of $D$ and $E(D)$ is called an arc set. Furthermore, an arc of the form $(u, u)$ is called a loop of $D$. If every arc of $D$ is expressed as $(u, v)$ where $u \neq v$, then $D$ is said to be a loopless digraph.

A digraph $D$ is called a complete digraph if for each $u, v \in V(D),(u, v) \in E(D)$. Moreover, the digraph $D$ is said to be semi-complete if for every $u, v \in V(D)$, $(u, v) \in E(D)$ or $(v, u) \in E(D)$. Furthermore, $D$ is said to be directed complete if it is loopless and for every $u, v \in V(D)$ with $u \neq v$, either $(u, v) \in E(D)$ or $(v, u) \in E(D)$.

Let $D$ be a digraph. Consider a sequence $P$ of distinct vertices in $V(D)$ as follows: 


$$
P:=v_{1}, v_{2}, \ldots, v_{k} \text { for some } k \in \mathbb{N} .
$$

If $P$ satisfies the condition that either $\left(v_{i}, v_{i+1}\right) \in E(D)$ or $\left(v_{i+1}, v_{i}\right) \in E(D)$ for all $i=1,2, \ldots, k-1$, then $P$ is said to be a semidipath from $v_{1}$ to $v_{k}$ in $D$. Moreover, if $P$ satisfies that $\left(v_{i}, v_{i+1}\right) \in E(D)$ for all $i=1,2, \ldots, k-1$, then $P$ is said to be a dipath from $v_{1}$ to $v_{k}$ in $D$. For convenience, throughout this paper, the notation $[u, v]$-semidipath ([u,v]-dipath) stands for the semidipath (dipath) from $u$ to $v$. For any two distinct vertices $u$ and $v$ in $V(D)$, a digraph $D$ ia said to be strongly connected if a $[u, v]$-dipath exists in $D$. Moreover, $D$ is said to be weakly connected if a $[u, v]$-semidipath exists in $D$. The digraph $D$ is said to be locally connected whenever a $[u, v]$-dipath exists in $D$, a $[v, u]$-dipath must exist in $D$ as well. In addition, $D$ is said to be unilaterally connected if either a $[u, v]$-dipath or a $[v, u]$-dipath exists in $D$. Furthermore, a maximal weakly connected subdigraph of $D$ will be called a component of $D$. A digraph $D$ is called an equivalence digraph if $E(D)$ is an equivalence relation on the set $V(D)$.

Recall that the Cayley digraph (simply called Cayley graph) Cay $(S, A)$ of a semigroup $S$ with respect to a connection set $A$ is a digraph with vertex set $V(\operatorname{Cay}(S, A))=S$ and $\operatorname{arc} \operatorname{set} E(\operatorname{Cay}(S, A))=\{(x, y) \in S \times S: y=x a$ for some $a \in$ $A\}$. The semigroup we study in this paper is $\operatorname{Fix}(X, Y)$, the semigroup of transformations with a fixed set. Further basic information and standard properties of $\operatorname{Fix}(X, Y)$ are described as follows.

Let $X$ be a set and $Y$ a nonempty subset of $X$. For convenience, we let $Y=$ $\left\{a_{i}: i \in I\right\}$ throughout this paper, unless otherwise stated. Hence for each $\alpha \in$ $\operatorname{Fix}(X, Y)$, we observe that $a_{i} \alpha=a_{i}$ for all $i \in I$. According to the convention presented in [7], we now give a presentation for the elements of $\operatorname{Fix}(X, Y)$. For each $\alpha \in \operatorname{Fix}(X, Y)$, we write

$$
\alpha=\left(\begin{array}{cc}
A_{i} & B_{j} \\
a_{i} & b_{j}
\end{array}\right)
$$

and take as understood that the subscripts $i$ and $j$ belong to the index sets $I$ and $J$, respectively. Moreover, $X \alpha$, the image of $\alpha$, is the disjoint union of $\left\{a_{i}: i \in I\right\}$ and $\left\{b_{j}: j \in J\right\}$ in which $a_{i} \alpha^{-1}=A_{i}$ and $b_{j} \alpha^{-1}=B_{j}$. Thus $A_{i} \cap Y=\left\{a_{i}\right\}$, $B_{j} \subseteq X \backslash Y$. Actually, the index set $J$ can be empty in general. Furthermore, the notation $\pi_{\alpha}$ stands for the set $\left\{x \alpha^{-1}: x \in X \alpha\right\}$.

Let $\mathscr{A}$ and $\mathscr{B}$ be families of sets. If for each $A \in \mathscr{A}$, there exists $B \in \mathscr{B}$ in which $A \subseteq B$, we say that $\mathscr{A}$ refines $\mathscr{B}$. It is not hard to prove the property stated in the following lemma. 
Lemma 2.1. Let $\alpha, \beta \in \operatorname{Fix}(X, Y)$. Then $\alpha=\beta \gamma$ for some $\gamma \in \operatorname{Fix}(X, Y)$ if and only if $\pi_{\beta}$ refines $\pi_{\alpha}$.

In this paper, we investigate the properties of the connectedness and the completeness of Cayley graphs $\operatorname{Cay}(\operatorname{Fix}(X, Y), A)$. Further, some structural properties of Cayley graphs of $\operatorname{Fix}(X, Y)$ related to minimal idempotents are considered in the next section.

\section{Some properties of Cayley graphs of $\operatorname{Fix}(X, Y)$ related to minimal idempotents}

Let $S$ be a semigroup. An element $e \in S$ is called an idempotent if $e^{2}=e$. The natural partial order on $E(S)$, the set of all idempotents on $S$, is defined by

$$
e \leq f \text { if and only if } e=e f=f e .
$$

An idempotent $e$ is said to be minimal if $e$ has the property $f \in E(S)$ and $f \leq e$ implies $f=e$.

Generally, it is well known that $\alpha \in T(X)$ is an idempotent if and only if $x \alpha=x$ for all $x \in X \alpha$. Consequently, we obtain that

$$
\alpha=\left(\begin{array}{cc}
A_{i} & B_{j} \\
a_{i} & b_{j}
\end{array}\right) \in \operatorname{Fix}(X, Y)
$$

is an idempotent if and only if $b_{j} \in B_{j}$ for all $j \in J$.

Further, let $E_{m}$ be a set of all minimal idempotents of $\operatorname{Fix}(X, Y)$. We can conclude from [7] that $E_{m}$ is not empty and it precisely contains all idempotents whose images coincide with $Y$, that is,

$$
E_{m}=\left\{\left(\begin{array}{c}
A_{i} \\
a_{i}
\end{array}\right):\left\{A_{i}: i \in I\right\} \text { is a partition of } X \text { with } a_{i} \in A_{i}\right\} .
$$

Moreover, we consider the property that how any minimal idempotent plays a role in the construction of the Cayley graph of $\operatorname{Fix}(X, Y)$ with respect to a connection set $A$.

First of all, we note that every minimal idempotent is a left zero element of $\operatorname{Fix}(X, Y)$ which is shown as follows. Let $\mu$ be a minimal idempotent in $\operatorname{Fix}(X, Y)$. Then $\mu$ is written as follows:

$$
\mu=\left(\begin{array}{c}
C_{i} \\
a_{i}
\end{array}\right)
$$

where $\left\{C_{i}: i \in I\right\}$ is a partition of $X$ and $a_{i} \in C_{i}$. Let $\alpha \in \operatorname{Fix}(X, Y)$. Thus we can write

$$
\alpha=\left(\begin{array}{ll}
A_{i} & B_{j} \\
a_{i} & b_{j}
\end{array}\right) .
$$


We observe that $\mu \alpha=\mu$, as required.

As the above consequence, we directly obtain that $\operatorname{Cay}(\operatorname{Fix}(X, Y), A)$ always contains loops since $(\mu, \mu) \in E(\operatorname{Cay}(\operatorname{Fix}(X, Y), A))$ for all $\mu \in E_{m}$.

Let $\alpha$ be any element in $\operatorname{Fix}(X, Y)$. We define

$$
N^{+}(\alpha)=\{\beta \in \operatorname{Fix}(X, Y): \beta \neq \alpha \text { and }(\alpha, \beta) \in E(\operatorname{Cay}(\operatorname{Fix}(X, Y), A))\} .
$$

As $\mu \in E_{m}$ is a left zero, all the arcs from $\mu$ will form loops and hence we have the following lemma.

Lemma 3.1. Let $A$ be a nonempty subset of $\operatorname{Fix}(X, Y)$ and $\mu$ a vertex of $\operatorname{Cay}(\operatorname{Fix}(X, Y), A)$. If $\mu \in E_{m}$, then $N^{+}(\mu)=\emptyset$.

We now describe the Cayley graph of $\operatorname{Fix}(X, Y)$ whose connection set is a singleton subset of $E_{m}$.

Theorem 3.2. Let $\left|E_{m}\right|=n$ for some $n \in \mathbb{N}$ and $A$ be a one-element-subset of $E_{m}$. Then $\operatorname{Cay}(\operatorname{Fix}(X, Y), A)$ is a disjoint union of $n$ subdigraphs which each of them contains exactly one minimal idempotent as its vertex.

Proof. Let $A=\{\mu\}$ for some $\mu \in E_{m}$. Let $C$ be a component of $\operatorname{Cay}(\operatorname{Fix}(X, Y), A)$ and $\alpha$ a vertex of $C$. Then we can write

$$
\alpha=\left(\begin{array}{ll}
A_{i} & B_{j} \\
a_{i} & b_{j}
\end{array}\right) .
$$

Since $\mu \in E_{m}, \mu$ is also expressed as

$$
\mu=\left(\begin{array}{c}
C_{i} \\
a_{i}
\end{array}\right) .
$$

For each $b_{j} \in X \alpha$, we have $b_{j} \in C_{k_{j}}$ for some $k_{j} \in I$. Let $K=\left\{k_{j} \in I: b_{j} \in\right.$ $C_{k_{j}}$ for some $\left.j \in J\right\}$ and $I^{\prime}=I \backslash K$. Then

$$
\alpha \mu=\left(\begin{array}{cc}
A_{i} & B_{j} \\
a_{i} & b_{j}
\end{array}\right)\left(\begin{array}{c}
C_{i} \\
a_{i}
\end{array}\right)=\left(\begin{array}{cc}
A_{i^{\prime}} & A_{k_{j}} \cup B_{j} \\
a_{i^{\prime}} & a_{k_{j}}
\end{array}\right) \in E_{m} .
$$

Since $(\alpha, \alpha \mu) \in E(\operatorname{Cay}(\operatorname{Fix}(X, Y), A))$, we obtain that $\alpha \mu$ lies in $C$. Hence $C$ contains a minimal idempotent $\alpha \mu$ as its vertex.

Next, we will show that $C$ contains exactly one minimal idempotent as its vertex. Suppose that $\mu_{1}$ and $\mu_{2}$ are different minimal idempotents contained in $V(C)$. Thus there exists a $\left[\mu_{1}, \mu_{2}\right]$-semidipath in $C$. Since $N^{+}\left(\mu_{1}\right)=\emptyset=N^{+}\left(\mu_{2}\right)$ by Lemma 3.1 , there exists a vertex $\alpha$ in which $\left(\alpha, \alpha_{1}\right)$ and $\left(\alpha, \alpha_{2}\right)$ are $\operatorname{arcs}$ of the $\left[\mu_{1}, \mu_{2}\right]$ semidipath. Hence the semidipath can be expressed as 


$$
\begin{array}{cll}
\stackrel{\alpha}{1}_{1} & \alpha & \alpha_{2} \\
\mu_{1}, \beta_{1}, \beta_{2}, \ldots, \beta_{s}, \beta_{s+1}, \beta_{s+2}, \beta_{s+3}, \beta_{s+4}, \ldots, \beta_{t}, \mu_{2}
\end{array}
$$

where $\left(\beta_{1}, \mu_{1}\right),\left(\beta_{t}, \mu_{2}\right),\left(\beta_{s+2}, \beta_{s+1}\right),\left(\beta_{s+2}, \beta_{s+3}\right)$ are arcs of the $\left[\mu_{1}, \mu_{2}\right]$-semidipath and either $\left(\beta_{i}, \beta_{i+1}\right)$ or $\left(\beta_{i+1}, \beta_{i}\right)$ is an arc of the $\left[\mu_{1}, \mu_{2}\right]$-semidipath for $1 \leq i \leq s$ and $s+3 \leq i \leq t-1$ for some $t \in \mathbb{N}$. By the definition of a semidipath, all vertices occurred in the above expression of the $\left[\mu_{1}, \mu_{2}\right]$-semidipath must be distinct. Since $\left(\alpha, \alpha_{1}\right),\left(\alpha, \alpha_{2}\right) \in E(\operatorname{Cay}(\operatorname{Fix}(X, Y), A))$, we have $\alpha_{1}=\alpha \mu$ and $\alpha_{2}=\alpha \mu$. It follows that $\alpha_{1}=\alpha_{2}$, this gives a contradiction. Therefore, there is precisely one minimal idempotent in $V(C)$.

Since each component of $\operatorname{Cay}(\operatorname{Fix}(X, Y), A)$ has exactly one minimal idempotent and a graph is a disjoint union of its components, we have the result.

According to the result stated in Theorem 3.2, we are interested to consider the structure of each component. The following results present some structural and algebraic properties of the component of $\operatorname{Cay}(\operatorname{Fix}(X, Y), A)$.

Corollary 3.3. Let $A=\{\mu\}$ for some $\mu \in E_{m}$ and $\eta \in E_{m}$ be arbitrary. If $C$ is the component of $\operatorname{Cay}(\operatorname{Fix}(X, Y), A)$ containing $\eta$, then $(\alpha, \eta) \in E(C)$ for all $\alpha \in V(C)$. Consequently, $(\alpha, \beta) \notin E(C)$ for all $\alpha, \beta \in V(C) \backslash\{\eta\}$.

Proof. Assume that $C$ is the component of $\operatorname{Cay}(\operatorname{Fix}(X, Y), A)$ containing $\eta$. For each $\alpha \in V(C)$, we have that $\alpha \mu$ is a minimal idempotent so that $\alpha \mu=\eta$. Hence any arc in $C$ is of the form $(\alpha, \eta)$.

The following example illustrates more clearly the above results, especially Theorem 3.2 and Corollary 3.3.

Example 3.4. Let $X=\{1,2,3,4\}$ and $Y=\{1,2\}$. Then $\operatorname{Fix}(X, Y)$ consists of 16 elements which each of them can be written as

$$
\alpha=\left(\begin{array}{llll}
1 & 2 & 3 & 4 \\
1 & 2 & p & q
\end{array}\right) \text { where } p, q \in X .
$$

For convenience, we will write $\alpha=(p q)$ in stead of writing $\alpha$ as above. We now let $A=\{(21)\}$ where $(21)$ is a minimal idempotent of $\operatorname{Fix}(X, Y)$. Hence the Cayley graph $\operatorname{Cay}(\operatorname{Fix}(X, Y), A)$ is shown in Figure 1. 


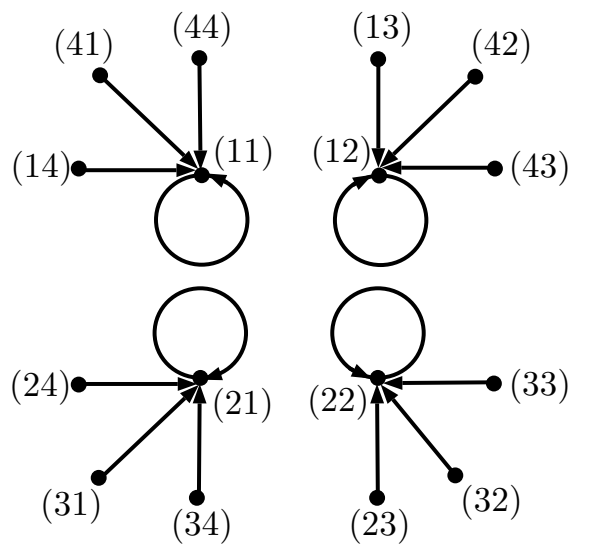

Figure 1. Cay $(\operatorname{Fix}(X, Y), A)$

Proposition 3.5. Let $A=\{\mu\}$ for some $\mu \in E_{m}$ and $\alpha \in \operatorname{Fix}(X, Y)$. Let $C$ be the component of $\operatorname{Cay}(\operatorname{Fix}(X, Y), A)$ containing a minimal idempotent $\eta$. Then $\alpha \in V(C)$ if and only if $\alpha$ satisfies the following conditions:

(i) $\pi_{\alpha}$ refines $\pi_{\eta}$;

(ii) for each $y \in X \alpha,\left(y \alpha^{-1}\right) \eta=\{y \mu\}$.

Proof. Assume that $\alpha \in V(C)$. By Corollary 3.3, we conclude that $(\alpha, \eta) \in E(C)$. Then $\eta=\alpha \mu$ and so $\pi_{\alpha}$ refines $\pi_{\eta}$ by Lemma 2.1. Next, let $y \in X \alpha$. We observe that $\left(y \alpha^{-1}\right) \eta=\left(y \alpha^{-1}\right) \alpha \mu=\{y \mu\}$.

Conversely, assume that the conditions hold. It is clear when $\alpha=\eta$. We consider when $\alpha \neq \eta$. From $\pi_{\alpha}$ refines $\pi_{\eta}$, we have by Lemma 2.1 that $\eta=\alpha \beta$ for some $\beta \in \operatorname{Fix}(X, Y)$. Let $x \in X$. Suppose that $x \alpha=y$ for some $y \in X$. Then $x \in y \alpha^{-1}$ which implies that $x \eta \in\left(y \alpha^{-1}\right) \eta=\{y \mu\}$ by the second condition. We get that $x \eta=y \mu$. Hence $x \alpha \beta=x \eta=y \mu=x \alpha \mu$, that is, $\alpha \beta=\alpha \mu$. Therefore, $\eta=\alpha \mu$ which leads to $(\alpha, \eta) \in E(\operatorname{Cay}(\operatorname{Fix}(X, Y), A))$. Since $\eta \in V(C)$, we conclude that $\alpha \in V(C)$.

\section{Connectedness of $\operatorname{Cay}(\operatorname{Fix}(X, Y), A)$}

In this section, we provide results on connectedness of $\operatorname{Cay}(\operatorname{Fix}(X, Y), A)$ consisting of the strongly connectedness, weakly connectedness, locally connectedness, and unilaterally connectedness.

Theorem 4.1. Let $A$ be a nonempty subset of $\operatorname{Fix}(X, Y)$. Then $\operatorname{Cay}(\operatorname{Fix}(X, Y), A)$ is strongly connected if and only if $X=Y$. 
Proof. Assume that $X=Y$. Clearly, $\operatorname{Fix}(X, Y)$ contains exactly one element, say $\alpha$, which directly implies that $A=\{\alpha\}$. Then $\operatorname{Cay}(\operatorname{Fix}(X, Y), A)$ will be as shown in Figure 2.

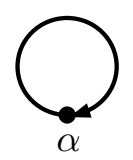

Figure 2. $\operatorname{Cay}(\{\alpha\},\{\alpha\})$

Hence $\operatorname{Cay}(\operatorname{Fix}(X, Y), A)$ is strongly connected.

Conversely, suppose that $Y$ is a proper subset of $X$. It is not hard to verify that $|\operatorname{Fix}(X, Y)| \geq 2$. For fixed $a_{i_{0}} \in Y$, let $I^{\prime}=I \backslash\left\{i_{0}\right\}$. Define

$$
\mu=\left(\begin{array}{cc}
X \backslash Y \cup\left\{a_{i_{0}}\right\} & a_{i^{\prime}} \\
a_{i_{0}} & a_{i^{\prime}}
\end{array}\right) .
$$

Then $\mu \in E_{m}$. By Lemma 3.1, we have $N^{+}(\mu)=\emptyset$. That means there is no arc joining from $\mu$ to any element in $\operatorname{Fix}(X, Y) \backslash\{\mu\}$. Consequently, there is no dipath from $\mu$ to another vertex. Therefore, $\operatorname{Cay}(\operatorname{Fix}(X, Y), A)$ is not strongly connected. This completes the proof.

Before we present the weakly connectedness of $\operatorname{Cay}(\operatorname{Fix}(X, Y), A)$, we need to prescribe the special notation as follows. Let $A$ be a nonempty subset of $\operatorname{Fix}(X, Y)$, the notation $A^{1}$ stands for the set $A$ adjoined the identity $i d_{X}$ of $\operatorname{Fix}(X, Y)$, that is,

$$
A^{1}= \begin{cases}A \cup\left\{i d_{X}\right\} & \text { if } i d_{X} \notin A ; \\ A & \text { if } i d_{X} \in A .\end{cases}
$$

Theorem 4.2. Let $A$ be a nonempty subset of $\operatorname{Fix}(X, Y)$. Then $\operatorname{Cay}(\operatorname{Fix}(X, Y), A)$ is weakly connected if and only if one of the following conditions holds:

(i) $X=Y$;

(ii) for each $\alpha, \beta \in \operatorname{Fix}(X, Y)$ where $\alpha \neq \beta$, there exist distinct elements $\alpha_{0}, \alpha_{1}, \alpha_{2}, \ldots, \alpha_{k+1} \in \operatorname{Fix}(X, Y)$ such that $\alpha_{i} A^{1} \cap \alpha_{i+1} A^{1} \neq \emptyset$ for all $i=0,1,2, \ldots, k$ where $\alpha_{0}=\alpha$ and $\alpha_{k+1}=\beta$.

Proof. Assume that $\operatorname{Cay}(\operatorname{Fix}(X, Y), A)$ is weakly connected. We now suppose that $Y$ is a proper subset of $X$. Then $|\operatorname{Fix}(X, Y)| \geq 2$. Next, let $\alpha, \beta \in \operatorname{Fix}(X, Y)$ be such that $\alpha \neq \beta$. By the weakly connectedness of $\operatorname{Cay}(\operatorname{Fix}(X, Y), A)$, there exists a sequence of vertices $\alpha_{1}, \alpha_{2}, \ldots, \alpha_{k}$ of $\operatorname{Cay}(\operatorname{Fix}(X, Y), A)$ in which 


$$
\alpha=\alpha_{0}, \alpha_{1}, \alpha_{2}, \ldots, \alpha_{k}, \alpha_{k+1}=\beta
$$

is a semidipath in $\operatorname{Cay}(\operatorname{Fix}(X, Y), A)$ and then those vertices together with $\alpha$ and $\beta$ are all distinct. Let $i \in\{0,1,2, \ldots, k\}$. By the property of the $[\alpha, \beta]$-semidipath expressed above, we obtain that $\left(\alpha_{i}, \alpha_{i+1}\right) \in E(\operatorname{Cay}(\operatorname{Fix}(X, Y), A))$ or $\left(\alpha_{i+1}, \alpha_{i}\right) \in$ $E(\operatorname{Cay}(\operatorname{Fix}(X, Y), A))$. Thus $\alpha_{i+1}=\alpha_{i} \lambda$ or $\alpha_{i}=\alpha_{i+1} \delta$ for some $\lambda, \delta \in A$. Hence $\alpha_{i+1} \in \alpha_{i+1} A^{1} \cap \alpha_{i} A^{1}$ or $\alpha_{i} \in \alpha_{i} A^{1} \cap \alpha_{i+1} A^{1}$, as required.

Conversely, if $X=Y$, then $\operatorname{Cay}(\operatorname{Fix}(X, Y), A)$ is weakly connected since it is strongly connected as shown in Theorem 4.1. Next, we assume that the second condition holds. Let $\alpha, \beta \in \operatorname{Fix}(X, Y)$ be such that $\alpha \neq \beta$. Then there exist distinct elements $\alpha_{0}, \alpha_{1}, \alpha_{2}, \ldots, \alpha_{k+1} \in \operatorname{Fix}(X, Y)$ such that $\alpha_{i} A^{1} \cap \alpha_{i+1} A^{1} \neq \emptyset$ for all $i=0,1,2, \ldots, k$ where $\alpha_{0}=\alpha$ and $\alpha_{k+1}=\beta$. Thus for each $i$, there exists $\lambda \in \alpha_{i} A^{1} \cap \alpha_{i+1} A^{1}$ which implies that $\alpha_{i} \delta=\lambda=\alpha_{i+1} \sigma$ for some $\delta, \sigma \in A^{1}$. We now have two possibilities to investigate.

Case 1: either $\delta$ or $\sigma$ is an identity. It is easily seen that there exists a directed edge joining between $\alpha_{i}$ and $\alpha_{i+1}$.

Case 2: neither $\delta$ nor $\sigma$ is an identity. Then $\left(\alpha_{i}, \lambda\right),\left(\alpha_{i+1}, \lambda\right) \in E(\operatorname{Cay}(\operatorname{Fix}(X, Y), A))$. Therefore, an $\left[\alpha_{i}, \alpha_{i+1}\right]$-semidipath exists in $\operatorname{Cay}(\operatorname{Fix}(X, Y), A)$.

From the above two cases, we can find an $[\alpha, \beta]$-semidipath in $\operatorname{Cay}(\operatorname{Fix}(X, Y), A)$, certainly. This completes the proof of the weakly connectedness of $\operatorname{Cay}(\operatorname{Fix}(X, Y), A)$.

The above theorem provides some necessary and sufficient conditions for $\operatorname{Cay}(\operatorname{Fix}(X, Y), A)$ being weakly connected which are quite general. To illustrate more clearly, we present another characterization of the weakly connectedness of $\operatorname{Cay}(\operatorname{Fix}(X, Y), A)$ where $X \backslash Y$ contains exactly one element as follows.

Theorem 4.3. Let $A$ be a nonempty subset of $\operatorname{Fix}(X, Y)$ where $|X \backslash Y|=1$. The following conditions are equivalent:

(i) $\operatorname{Cay}(\operatorname{Fix}(X, Y), A)$ is weakly connected;

(ii) $Y \subseteq \bigcup_{\alpha \in A}(X \backslash Y) \alpha$;

(iii) $E_{m} \subseteq A$.

Proof. Let $A$ be a nonempty subset of $\operatorname{Fix}(X, Y)$ where $|X \backslash Y|=1$. Assume that $X \backslash Y=\{b\}$.

(i) $\Rightarrow$ (ii) Let $\operatorname{Cay}(\operatorname{Fix}(X, Y), A)$ be weakly connected. Further, let $a_{i} \in Y$ and $I^{\prime}=I \backslash\{i\}$. Define $\mu \in \operatorname{Fix}(X, Y)$ by

$$
\mu=\left(\begin{array}{cc}
\left\{a_{i}, b\right\} & a_{i^{\prime}} \\
a_{i} & a_{i^{\prime}}
\end{array}\right) .
$$


Then $\mu \in E_{m}$. It follows that $N^{+}(\mu)=\emptyset$ by Lemma 3.1. Since Cay $(\operatorname{Fix}(X, Y), A)$ is weakly connected, there exists $\beta \in \operatorname{Fix}(X, Y) \backslash\{\mu\}$ such that $(\beta, \mu) \in$ $E(\operatorname{Cay}(\operatorname{Fix}(X, Y), A))$. That means $\mu=\beta \gamma$ for some $\gamma \in A$ and so $\mu \in N^{+}(\beta)$. By the contraposition of Lemma 3.1, we conclude that $\beta$ is not a minimal idempotent. It follows that $b \beta \notin Y$, that is, $b \beta=b$. Therefore, $a_{i}=b \mu=b \beta \gamma=b \gamma \in(X \backslash Y) \gamma \subseteq$ $\bigcup_{\alpha \in A}(X \backslash Y) \alpha$. Thus $Y \subseteq \bigcup_{\alpha \in A}(X \backslash Y) \alpha$.

(ii) $\Rightarrow$ (iii) Assume that $Y \subseteq \bigcup_{\alpha \in A}(X \backslash Y) \alpha$. Let $\mu \in E_{m}$. Then $b \mu \in Y \subseteq$ $\bigcup_{\alpha \in A}(X \backslash Y) \alpha$ which implies that $b \mu \in(X \backslash Y) \beta$ for some $\beta \in A$. Hence $b \mu=b \beta$. Since $a \mu=a=a \beta$ for all $a \in Y$ and we have $b \mu=b \beta$, it follows that $\mu=\beta \in A$ which leads to $E_{m} \subseteq A$.

(iii) $\Rightarrow$ (i) Let $E_{m}$ be a subset of $A$ and $\alpha, \beta \in \operatorname{Fix}(X, Y)$. It is obvious that $\alpha \mu_{1}=\eta_{1}$ and $\beta \mu_{2}=\eta_{2}$ for some $\mu_{1}, \mu_{2}, \eta_{1}, \eta_{2} \in E_{m} \subseteq A$. Hence $\left(\alpha, \eta_{1}\right),\left(\beta, \eta_{2}\right) \in$ $E(\operatorname{Cay}(\operatorname{Fix}(X, Y), A))$. Moreover, we have $\left(i d_{X}, \eta_{1}\right),\left(i d_{X}, \eta_{2}\right) \in E(\operatorname{Cay}(\operatorname{Fix}(X, Y), A))$. Therefore, we can find an $[\alpha, \beta]$-semidipath in $\operatorname{Cay}(\operatorname{Fix}(X, Y), A)$ which implies that $\operatorname{Cay}(\operatorname{Fix}(X, Y), A)$ is weakly connected.

For characterizing the locally connectedness of $\operatorname{Cay}(\operatorname{Fix}(X, Y), A)$, we need the following lemma.

Lemma 4.4. Let $A$ be a nonempty subset of $\operatorname{Fix}(X, Y)$ and $\alpha, \beta \in \operatorname{Fix}(X, Y)$. If there exists a dipath joining from $\alpha$ to $\beta$ in $\operatorname{Cay}(\operatorname{Fix}(X, Y), A)$, then $\pi_{\alpha}$ refines $\pi_{\beta}$.

Proof. Assume that there exists a dipath $P$ joining from $\alpha$ to $\beta$ in $\operatorname{Cay}(\operatorname{Fix}(X, Y), A)$. Thus $P$ can be expressed as a sequence $\alpha, \gamma_{1}, \gamma_{2}, \ldots, \gamma_{k}, \beta$ of some vertices of $\operatorname{Cay}(\operatorname{Fix}(X, Y), A)$. Hence $\beta=\gamma_{k} \lambda_{1}=\left(\gamma_{k-1} \lambda_{2}\right) \lambda_{1}=\ldots=\left(\gamma_{1} \lambda_{k}\right) \lambda_{k-1} \cdots \lambda_{2} \lambda_{1}=$ $\alpha \lambda_{k+1} \lambda_{k} \cdots \lambda_{2} \lambda_{1}$ where $\lambda_{i} \in A$ for all $i=1,2, \ldots, k+1$. Since $\lambda_{k+1} \lambda_{k} \cdots \lambda_{2} \lambda_{1} \in$ $\operatorname{Fix}(X, Y)$, we obtain that $\pi_{\alpha}$ refines $\pi_{\beta}$ by Lemma 2.1. This completes the proof.

Further, the notation $H_{i d_{X}}$ means an equivalence $\mathcal{H}$-class containing $i d_{X}$ and elements in $\operatorname{Fix}(X, Y)$ which $\mathcal{H}$-relate to $i d_{X}$ where $\mathcal{H}$ is one of Green's relations (see [8]). Moreover, it is well known that $H_{i d_{X}}$ is a group of all bijections, exactly.

Theorem 4.5. Let $A$ be a nonempty subset of $\operatorname{Fix}(X, Y)$. Then $\operatorname{Cay}(\operatorname{Fix}(X, Y), A)$ is locally connected if and only if $A \subseteq H_{i d_{X}}$.

Proof. Assume that $\operatorname{Cay}(\operatorname{Fix}(X, Y), A)$ is locally connected. We first let $\alpha \in A$. Then $\left(i d_{X}, \alpha\right) \in E(\operatorname{Cay}(\operatorname{Fix}(X, Y), A))$. By the locally connectedness of $\operatorname{Cay}(\operatorname{Fix}(X, Y), A)$, it follows that there must be an $\left[\alpha, i d_{X}\right]$-dipath in $\operatorname{Cay}(\operatorname{Fix}(X, Y), A)$. By Lemma 
4.4, we can conclude that $\pi_{\alpha}$ refines $\pi_{i d_{X}}$ and so $\alpha$ is injective. Since $X$ is finite, we obtain that $\alpha$ is a bijection, that is, $\alpha \in H_{i d_{X}}$. Hence $A \subseteq H_{i d_{X}}$, as required.

Conversely, assume that $A \subseteq H_{i d_{X}}$. Let $\alpha, \beta \in \operatorname{Fix}(X, Y)$ be such that $\operatorname{Cay}(\operatorname{Fix}(X, Y), A)$ contains an $[\alpha, \beta]$-dipath. Then there exist bijections $\lambda_{1}, \lambda_{2}, \ldots, \lambda_{k} \in A \subseteq H_{i d_{X}}$ for some $k \in \mathbb{N}$ in which $\beta=\alpha \lambda_{1} \lambda_{2} \cdots \lambda_{k}$. Without loss of generality, we may assume that such $\lambda_{i}$ is not an identity of $\operatorname{Fix}(X, Y)$ for all $i=1,2, \ldots, k$. Since $H_{i d_{X}}$ is a finite group, we can define the order of $\lambda_{i}$ as $r_{i}$ where $r_{i}>1$ for all $i=1,2, \ldots, k$, that is, $\lambda_{i}^{r_{i}}$ is an identity of $H_{i d_{X}}$ which coincides with the identity of $\operatorname{Fix}(X, Y)$. Hence

$$
\alpha=\beta \lambda_{k}^{r_{k}-1} \cdots \lambda_{2}^{r_{2}-1} \lambda_{1}^{r_{1}-1} \text { where } \lambda_{1}, \lambda_{2}, \ldots, \lambda_{k} \in A .
$$

This ensures that there exists a dipath from $\beta$ to $\alpha$. So $\operatorname{Cay}(\operatorname{Fix}(X, Y), A)$ is locally connected.

Theorem 4.6. Let $A$ be a nonempty subset of $\operatorname{Fix}(X, Y)$. Then $\operatorname{Cay}(\operatorname{Fix}(X, Y), A)$ is unilaterally connected if and only if one of the following conditions holds:

(i) $X=Y$;

(ii) $|X|=2,|Y|=1$ and $A$ contains a minimal idempotent.

Proof. Let $\operatorname{Cay}(\operatorname{Fix}(X, Y), A)$ be unilaterally connected. Suppose that $Y$ is a proper subset of $X$. We first prove that $|Y|=1$ by supposing that this is false, so $|Y| \geq 2$. Clearly, $\operatorname{Fix}(X, Y)$ contains at least two minimal idempotents. Let $\mu_{1}, \mu_{2} \in E_{m}$. Then by Lemma 3.1, we conclude that $N^{+}\left(\mu_{1}\right)=\emptyset=N^{+}\left(\mu_{2}\right)$. We consequently obtain that there is no dipath joining between $\mu_{1}$ and $\mu_{2}$ which contradicts to the unilaterally connectedness of $\operatorname{Cay}(\operatorname{Fix}(X, Y), A)$. Thus $|Y|=1$. Next, we suppose that $|X| \geq 3$. Let $Y=\{a\}$. Choose $b_{1}, b_{2} \in X \backslash Y$ in which $b_{1} \neq b_{2}$. Define

$$
\alpha=\left(\begin{array}{cc}
\left\{a, b_{1}\right\} & X \backslash\left\{a, b_{1}\right\} \\
a & b_{1}
\end{array}\right) \text { and } \beta=\left(\begin{array}{cc}
\left\{a, b_{2}\right\} & X \backslash\left\{a, b_{2}\right\} \\
a & b_{1}
\end{array}\right) .
$$

Then $\alpha, \beta \in \operatorname{Fix}(X, Y)$. Moreover, $\pi_{\alpha}=\left\{\left\{a, b_{1}\right\}, X \backslash\left\{a, b_{1}\right\}\right\}$ and $\pi_{\beta}=\left\{\left\{a, b_{2}\right\}\right.$, $\left.X \backslash\left\{a, b_{2}\right\}\right\}$ such that they do not mutually refine. By Lemma 4.4, there is no dipath joining them which again contradicts to the unilaterally connectedness of $\operatorname{Cay}(\operatorname{Fix}(X, Y), A)$. Therefore, $|X|=2$. For convenience, we may assume that $X=\{a, b\}$ and $Y=\{a\}$. Then $\operatorname{Fix}(X, Y)$ contains exactly two elements

$$
\gamma=\left(\begin{array}{ll}
a & b \\
a & a
\end{array}\right) \text { and } i d_{X}=\left(\begin{array}{ll}
a & b \\
a & b
\end{array}\right)
$$

where $\gamma$ is the unique minimal idempotent of $\operatorname{Fix}(X, Y)$. 
Suppose that $A$ does not contain a minimal idempotent. So $A=\left\{i d_{X}\right\}$ and $\operatorname{Cay}(\operatorname{Fix}(X, Y), A)$ is shown in Figure 3. We observe that $\operatorname{Cay}(\operatorname{Fix}(X, Y), A)$ is not unilaterally connected which is impossible. Hence the connection set $A$ of $\operatorname{Cay}(\operatorname{Fix}(X, Y), A)$ must contain a minimal idempotent. Therefore, the necessity is completely proved.
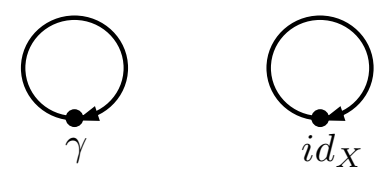

Figure 3. $\operatorname{Cay}(\operatorname{Fix}(X, Y), A)$

Conversely, assume that $\operatorname{Cay}(\operatorname{Fix}(X, Y), A)$ satisfies one of such sufficient conditions. If the first condition holds for $\operatorname{Cay}(\operatorname{Fix}(X, Y), A)$, then $\operatorname{Cay}(\operatorname{Fix}(X, Y), A)$ is strongly connected by Theorem 4.1 which consequently leads to the unilaterally connectedness of $\operatorname{Cay}(\operatorname{Fix}(X, Y), A)$. Hence we suffice to consider the case when $\operatorname{Cay}(\operatorname{Fix}(X, Y), A)$ satisfies the second condition. Conveniently, we take $X=\{a, b\}$ and $Y=\{a\}$. Thus $\operatorname{Fix}(X, Y)$ contains exactly two elements $\gamma$ and $i d_{X}$ defined in the proof of the necessity. As the connection set $A$ contains a minimal idempotent, we conclude that there are two possibilities for such a connection set, that is, $A$ can be $\{\gamma\}$ or $\left\{\gamma, i d_{X}\right\}$. To illustrate more explicit, let $A_{1}=\{\gamma\}$ and $A_{2}=\left\{\gamma, i d_{X}\right\}$. Then Cayley graphs of $\operatorname{Fix}(X, Y)$ with respect to connection sets $A_{1}$ and $A_{2}$ are shown in Figures 4 and 5, respectively.

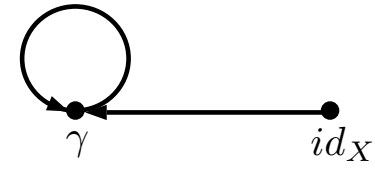

Figure 4. $\operatorname{Cay}\left(\operatorname{Fix}(X, Y), A_{1}\right)$

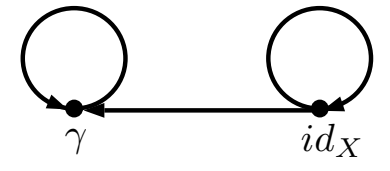

Figure 5. $\operatorname{Cay}\left(\operatorname{Fix}(X, Y), A_{2}\right)$

Clearly, both Cayley graphs are unilaterally connected.

\section{Completeness of $\operatorname{Cay}(\mathbf{F i x}(X, Y), A)$}

In this section, we study characterizations of the completeness of $\operatorname{Cay}(\operatorname{Fix}(X, Y), A)$. Besides the completeness of $\operatorname{Cay}(\operatorname{Fix}(X, Y), A)$, the concept of an equivalence digraph is also considered for $\operatorname{Cay}(\operatorname{Fix}(X, Y), A)$. We now present the following results. 
Theorem 5.1. Let $A$ be a nonempty subset of $\operatorname{Fix}(X, Y)$. Then $\operatorname{Cay}(\operatorname{Fix}(X, Y), A)$ is complete if and only if $X=Y$.

Proof. It is obvious that $\operatorname{Cay}(\operatorname{Fix}(X, Y), A)$ is complete whenever $X=Y$ since it contains exactly one vertex and a loop attached to the vertex.

To prove the converse, assume that $\operatorname{Cay}(\operatorname{Fix}(X, Y), A)$ is complete. Suppose to the contrary that there exists $b \in X \backslash Y$. Choose $a_{i_{0}} \in Y$ and let $I^{\prime}=I \backslash\left\{i_{0}\right\}$. Define $\alpha, \beta \in \operatorname{Fix}(X, Y)$ by

$$
\alpha=\left(\begin{array}{cc}
X \backslash Y \cup\left\{a_{i_{0}}\right\} & a_{i^{\prime}} \\
a_{i_{0}} & a_{i^{\prime}}
\end{array}\right) \text { and } \beta=\left(\begin{array}{cc}
a_{i} & X \backslash Y \\
a_{i} & b
\end{array}\right) .
$$

Clearly, $\alpha \in E_{m}$ and $\beta \notin E_{m}$. By the completeness of $\operatorname{Cay}(\operatorname{Fix}(X, Y), A)$, we have $(\alpha, \beta),(\beta, \alpha) \in E(\operatorname{Cay}(\operatorname{Fix}(X, Y), A))$. The edge $(\alpha, \beta)$ implies that there exists $\lambda \in A$ in which $\beta=\alpha \lambda=\alpha$ since $\alpha \in E_{m}$. This gives a contradiction. So we can conclude that $X=Y$.

Theorem 5.2. Let $A$ be a nonempty subset of $\operatorname{Fix}(X, Y)$. Then $\operatorname{Cay}(\operatorname{Fix}(X, Y), A)$ is semi-complete if and only if one of the following conditions holds:

(i) $X=Y$;

(ii) $|X|=2,|Y|=1$ and $A=\operatorname{Fix}(X, Y)$.

Proof. Let $\operatorname{Cay}(\operatorname{Fix}(X, Y), A)$ be semi-complete. Suppose that $Y$ is a proper subset of $X$. Similarly, we can apply the proof of the necessity of Theorem 4.6 to conclude that $|X|=2$ and $|Y|=1$. Consequently, $\operatorname{Fix}(X, Y)=\left\{i d_{X}, \gamma\right\}$ and $\gamma \in E_{m}$. If $i d_{X} \notin A$, then $\left(i d_{X}, i d_{X}\right) \notin E(\operatorname{Cay}(\operatorname{Fix}(X, Y), A))$ or if $\gamma \notin$ $A$, then $\left(\gamma, i d_{X}\right),\left(i d_{X}, \gamma\right) \notin E(\operatorname{Cay}(\operatorname{Fix}(X, Y), A))$, which contradicts the semicompleteness of $\operatorname{Cay}(\operatorname{Fix}(X, Y), A)$. Hence both of $i d_{X}$ and $\gamma$ must belong to $A$ which implies that $A=\operatorname{Fix}(X, Y)$.

Conversely, assume that the second condition holds. Thus $\operatorname{Cay}(\operatorname{Fix}(X, Y), A)$ can be drawn as Figure 5 which is shown in the proof of the sufficiency of Theorem 4.6. So $\operatorname{Cay}(\operatorname{Fix}(X, Y), A)$ is semi-complete. Moreover, the assertion is clear whenever $X=Y$.

Note that $\operatorname{Fix}(X, Y)$ always contains minimal idempotents. Since they are left zeros of $\operatorname{Fix}(X, Y)$, the digraph $\operatorname{Cay}(\operatorname{Fix}(X, Y), A)$ always contains loops attached to the minimal idempotents. Consequently, $\operatorname{Cay}(\operatorname{Fix}(X, Y), A)$ is not a loopless digraph. Hence, we have the following theorem.

Theorem 5.3. Let $A$ be a nonempty subset of $\operatorname{Fix}(X, Y)$. Then $\operatorname{Cay}(\operatorname{Fix}(X, Y), A)$ is never a directed complete digraph. 
We now present the following results on an equivalence digraph for $\operatorname{Cay}(\operatorname{Fix}(X, Y), A)$.

Lemma 5.4. Let $A$ be a nonempty subset of $\operatorname{Fix}(X, Y)$. Then $E(\operatorname{Cay}(\operatorname{Fix}(X, Y), A))$ is reflexive if and only if $i d_{X} \in A$.

Proof. Let $E(\operatorname{Cay}(\operatorname{Fix}(X, Y), A))$ be a reflexive relation. Since $\operatorname{Fix}(X, Y)$ contains the identity $i d_{X}$, we obtain that $\left(i d_{X}, i d_{X}\right) \in E(\operatorname{Cay}(\operatorname{Fix}(X, Y), A))$. Thus there exists $\alpha \in A$ such that $i d_{X}=i d_{X} \alpha=\alpha \in A$.

For the converse, it is easy to verify that $(\alpha, \alpha) \in E(\operatorname{Cay}(\operatorname{Fix}(X, Y), A))$ for all $\alpha \in \operatorname{Fix}(X, Y)$ whenever $A$ contains an identity of $\operatorname{Fix}(X, Y)$. We obtain that $E(\operatorname{Cay}(\operatorname{Fix}(X, Y), A))$ is reflexive, as required.

Recall that $H_{i d_{X}}$ is a group with identity $i d_{X}$. For a nonempty subset $B$ of $H_{i d_{X}}$, the notation $B^{-1}$ stands for the set $\left\{\delta^{-1}: \delta \in B\right\}$ which is useful for proving the symmetry of $E(\operatorname{Cay}(\operatorname{Fix}(X, Y), A))$ stated in the following lemma.

Lemma 5.5. Let $A$ be a nonempty subset of $\operatorname{Fix}(X, Y)$. Then $E(\operatorname{Cay}(\operatorname{Fix}(X, Y), A))$ is symmetric if and only if $A \subseteq H_{i d_{X}}$ and $A=A^{-1}$.

Proof. Assume that $E(\operatorname{Cay}(\operatorname{Fix}(X, Y), A))$ is symmetric. Clearly, $\operatorname{Cay}(\operatorname{Fix}(X, Y), A)$ is locally connected. By Theorem 4.5, we have $A \subseteq H_{i d_{X}}$. Further, let $\alpha \in A$. Then $\left(i d_{X}, \alpha\right) \in E(\operatorname{Cay}(\operatorname{Fix}(X, Y), A))$. By the assumption, we get that $\left(\alpha, i d_{X}\right) \in$ $E(\operatorname{Cay}(\operatorname{Fix}(X, Y), A))$, that is, $i d_{X}=\alpha \beta$ for some $\beta \in A$. Therefore, $\beta \in H_{i d_{X}}$ and $\alpha=\beta^{-1} \in A^{-1}$. On the other hand, let $\gamma^{-1} \in A^{-1}$. Thus $\gamma \in A$. By the same argument, we conclude that $\gamma \in A^{-1}$. This directly implies that $\gamma^{-1} \in A$. Consequently, we have $A=A^{-1}$.

Conversely, assume that $A \subseteq H_{i d_{X}}$ and $A=A^{-1}$. Let $\alpha, \beta \in \operatorname{Fix}(X, Y)$ be such that $(\alpha, \beta) \in E(\operatorname{Cay}(\operatorname{Fix}(X, Y), A))$. Then $\beta=\alpha \gamma$ for some $\gamma \in A$. Since $A \subseteq H_{i d_{X}}$, we obtain that $\gamma^{-1}$ exists in $H_{i d_{X}}$ and $\gamma^{-1} \in A^{-1}=A$. As the fact that $\alpha=\beta \gamma^{-1}$ where $\gamma^{-1} \in A$, we conclude that $(\beta, \alpha) \in E(\operatorname{Cay}(\operatorname{Fix}(X, Y), A))$. This implies that $E(\operatorname{Cay}(\operatorname{Fix}(X, Y), A))$ is symmetric.

Lemma 5.6. Let $A$ be a nonempty subset of $\operatorname{Fix}(X, Y)$. Then $E(\operatorname{Cay}(\operatorname{Fix}(X, Y), A))$ is transitive if and only if $A$ is a subsemigroup of $\operatorname{Fix}(X, Y)$.

Proof. Assume that $E(\operatorname{Cay}(\operatorname{Fix}(X, Y), A))$ is transitive. Let $\alpha, \beta \in A$. Clearly, we have $\left(i d_{X}, \alpha\right),(\alpha, \alpha \beta) \in E(\operatorname{Cay}(\operatorname{Fix}(X, Y), A))$. Since $E(\operatorname{Cay}(\operatorname{Fix}(X, Y), A))$ is transitive, we obtain that $\left(i d_{X}, \alpha \beta\right) \in E(\operatorname{Cay}(\operatorname{Fix}(X, Y), A))$ which leads to $\alpha \beta=$ $i d_{X} \gamma=\gamma$ for some $\gamma \in A$. Hence $\alpha \beta \in A$. Consequently, $A$ is a subsemigroup of $\operatorname{Fix}(X, Y)$. 
Conversely, let $A$ be a subsemigroup of $\operatorname{Fix}(X, Y)$. Further, let $\alpha, \beta, \gamma \in \operatorname{Fix}(X, Y)$ in which $(\alpha, \beta),(\beta, \gamma) \in E(\operatorname{Cay}(\operatorname{Fix}(X, Y), A))$. Thus there exist $\lambda, \mu \in A$ such that $\beta=\alpha \lambda$ and $\gamma=\beta \mu$, respectively. It follows that $\gamma=\beta \mu=\alpha \lambda \mu$. Since $A$ is a subsemigroup of $\operatorname{Fix}(X, Y)$, we have $\lambda \mu \in A$. This implies that $(\alpha, \gamma) \in$ $E(\operatorname{Cay}(\operatorname{Fix}(X, Y), A))$. Therefore, $E(\operatorname{Cay}(\operatorname{Fix}(X, Y), A))$ is transitive.

From Lemmas 5.4, 5.5 and 5.6, we directly have the following theorem.

Theorem 5.7. Let $A$ be a nonempty subset of $\operatorname{Fix}(X, Y)$. Then $\operatorname{Cay}(\operatorname{Fix}(X, Y), A)$ is an equivalence digraph if and only if $A$ is a subgroup of $H_{i d_{X}}$.

Acknowledgement. The authors are grateful to the referee(s) for corrections and valuable suggestions on the manuscript. This research was supported by Khon Kaen University.

\section{References}

[1] M. Afkhami, H. Reza Barani, K. Khashyarmanesh and F. Rahbarnia, A new class of Cayley graphs, J. Algebra Appl., 15(4) (2016), 1650076 (8 pp).

[2] Y. Chaiya, P. Honyam and J. Sanwong, Natural partial orders on transformation semigroups with fixed sets, Int. J. Math. Math. Sci., (2016), 2759090 (7 $\mathrm{pp})$.

[3] A. H. Clifford and G. B. Preston, The Algebraic Theory of Semigroups, Vol. I, Mathematical Surveys, 7, American Mathematical Society, Providence, R.I., 1961.

[4] A. H. Clifford and G. B. Preston, The Algebraic Theory of Semigroups, Vol. II, Mathematical Surveys, 7, American Mathematical Society, Providence, R.I., 1967.

[5] S. Fan and Y. Zeng, On Cayley graphs of bands, Semigroup Forum, 74(1) (2007), 99-105.

[6] Y. Hao and Y. Luo, On the Cayley graphs of left (right) groups, Southeast Asian Bull. Math., 34(4) (2010), 685-691.

[7] P. Honyam and J. Sanwong, Semigroups of transformations with fixed sets, Quaest. Math., 36(1) (2013), 79-92.

[8] J. M. Howie, Fundamentals of Semigroup Theory, London Mathematical Society Monographs, New Series, 12, Oxford Science Publications, The Clarendon Press, Oxford University Press, New York, 1995.

[9] A. V. Kelarev, On Cayley graphs of inverse semigroups, Semigroup Forum, 72(3) (2006), 411-418. 
[10] B. Khosravi and M. Mahmoudi, On Cayley graphs of rectangular groups, Discrete Math., 310(4) (2010), 804-811.

[11] Y. Luo, Y. Hao and G. T. Clarke, On the Cayley graphs of completely simple semigroups, Semigroup Forum, 82(2) (2011), 288-295.

[12] R. P. Panda and K. V. Krishna, On connectedness of power graphs of finite groups, J. Algebra Appl., 17(10) (2018), 1850184 (20 pp).

[13] S. Pirzada, An Introduction to Graph Theory, Universities Press, Orient Blackswan, India, 2012.

[14] A. Riyas and K. Geetha, Some properties of Cayley graphs of full transformation semigroups, Int. J. Math. Archive, 9(4) (2018), 64-69.

[15] D. Sinha and D. Sharma, Structural properties of absorption Cayley graphs, Appl. Math. Inf. Sci., 10(6) (2016), 2237-2245.

[16] T. Suksumran and S. Panma, On connected Cayley graphs of semigroups, Thai J. Math., 13(3) (2015), 641-652.

[17] C. Tisklang and S. Panma, On connectedness of Cayley graphs of finite transformation semigroups, Thai J. Math., Special issue (2018), 261-271.

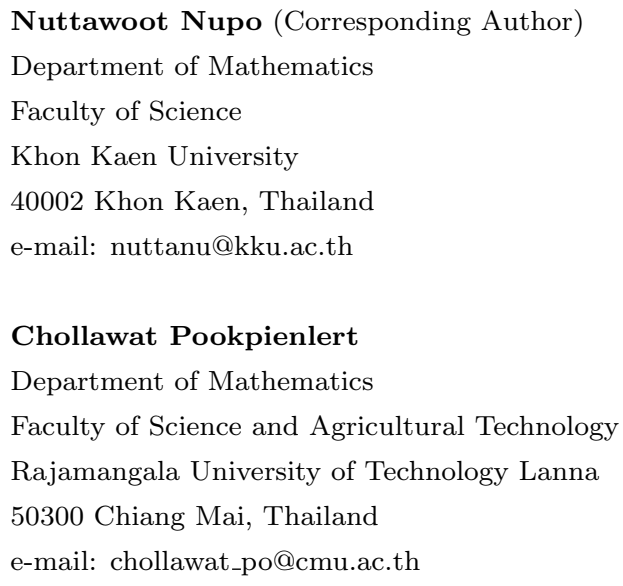

